\title{
Maxillofacial Radiology 180
}

SADJ May 2020, Vol. 75 No. 4 p218

CJ Nortjé

Figures, A \& B are images of a patient who presented with underlying fractures of the body on the right side and angle of the mandible on the left side. Discuss the most important radiological features discernible on the radiographs and what are your conclusions?
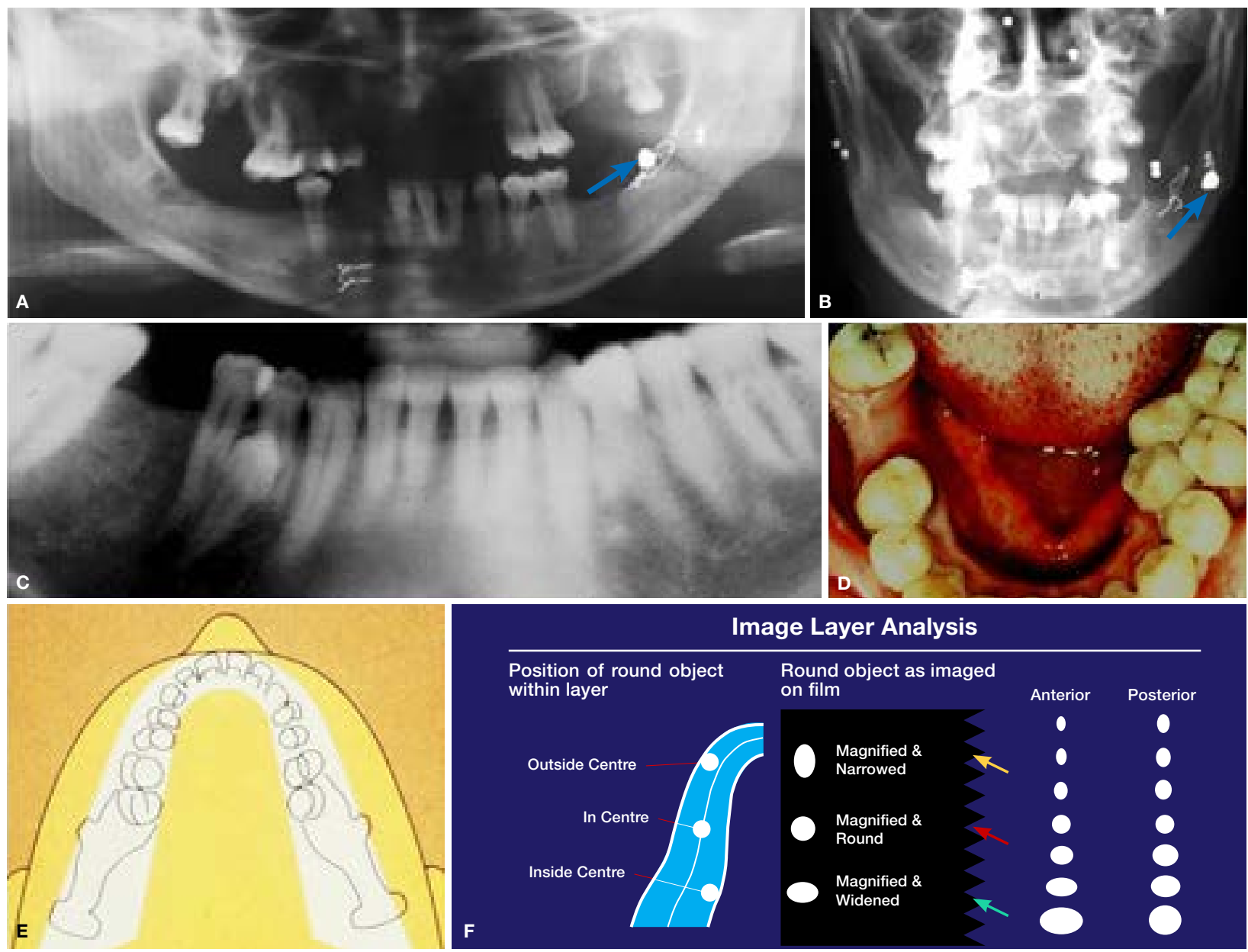

\section{INTERPRETATION}

The most important findings are: Figure A shows ligature wiring of the bilateral fractures and the presence of a pellet (blue arrow). However the posterior-anterior mandible radiograph (Fig. B) of the same patient shows multiple pellets which are not discernible on the pantomograph (Fig. A). The blue arrow in Figure A represents the same blue arrow in Figure B. To illustrate to you how easy it is to have a problem I want to illustrate the following case. Looking at the cropped pantomograph (Fig. C) we see a missing tooth in the right lower jaw, as well as a supernumerary tooth. But if you examine the patient clinically, you will notice that there are two supernumerary teeth (Fig. D) in the lower left mandible, which is not depicted on the radiograph because the supernumerary teeth are not present in the layer (focal trough) (Fig. E).

Christoffel J Nortjé: $B C h D, P h D, A B O M R, D S c$. Faculty of Dentistry, University of the Western Cape.

ORCID Number: 0000-0002-9717-5514

Email: cnortje@uwc.ac.za
What it means is that a small error in the positioning of the patient may cause diagnostic problems for example if it gets close to the edge of the layer or when the structure is not exactly in the middle of the layer, may result in misdiagnosis. If something is not in the layer, you would not be able to observe it. The many birdshot present in Figure $\mathrm{B}$, are not discernible in the pantomograph because the birdshot was not in the layer. However when something is in the middle of the layer it has its nice configuration and a perfect shape. Looking at Figure $F$ you will notice that the metal ball has a perfectly round shape if it is in the centre of the layer (red arrow). However if the object is close to the edge of the layer towards the film (buccally) you will notice that it is narrow (yellow arrow) and if the object is on lingual side of the layer, the object appears to be widened (green arrow).

\section{Reference}

1. Langlais RP, Langland OE, Nortje CJ: Diagnostic Imaging of the Jaws. $1^{\text {st }}$ edition. Williams \& Wilkens. 1995; 225 - 65. 